\title{
Application of a Higher RPA to a Model $\pi$-Electron System
}

\author{
Tai-ichi Shibuya and Vincext McKoy \\ Arthur A mos Noyes Laboratory of Chemical Physics, ${ }^{*}$ California Institute of Technology, Pasadena, California
}

(Received 11 May 1970)

\begin{abstract}
We have applied a proposed higher-order random phase approximation (RPA) to the simple model system of two $\pi$ electrons in the double bond of ethylene. The higher-order RPA removes some difficulties involved in the usual RPA, but retains the form of the RPA equations. To derive the higher RPA we retain all terms linear in the ground state correlation coefficients in the equations of motion for the excitation energy and in the equation determining the ground state. These equations are solved self-consistently and are simpler to handle than a configuration interaction solution in a realistic example. We do not use the quasiboson approximation.
\end{abstract}

\section{INTRODUCTION}

A major problem in the study of the electronic structure of atoms and molecules is the role of correlations, particularly for observables other than the total energy. Two such properties of interest are the excitation energies and oscillator strengths of transitions to low-lying excited electronic states. Although the general features of an electronic excitation can usually be understood in terms of a simple model, e.g., HartreeFock orbital description, a more accurate description can involve considerable effort. A simple example would be to attempt to improve the Hartree-Fock estimate of an excitation energy by carrying out an extensive configuration interaction study on both the ground and excited states separately. For a reasonable mumber of electrons this approach can rapidly get out of hand before the results are significantly improved.

A more direct approach to predicting excitation energies is the random phase approximation (RPA). The RPA method calculates excitation energies directly, treating the stationary state wavefunctions as of secondary importance. The RPA equations can be derived in a variety of ways. Rowe ${ }^{1}$ has reviewed these methods and has also proposed a new equations-ofmotion method for studying the excited states of a system relative to its correlated ground state. The equations of motion are expressed as the ground state expectation of operator equations. The simplest solution to these equations, equivalent to the RPA, is obtained by considering only single particle-hole excited states, i.e., singly excited relative to the ground state, and by approximating the ground state wavefunction in the expectation value by the Hartree-Fock particle-hole vacuum. We have used the RPA method to study the low-lying excited states of ethylene and formaldehyde. ${ }^{2}$ These results showed some promising features of the method, in particular, in predicting excitation energies and transition moments for singlet excited states. However, the method works poorly for triplet excited states, yielding imaginary frequencies for some transitions. We have developed a higher-order $\mathrm{RPA}^{3}$ which removes these difficulties but yet formally retains the simple form of the RPA equations. We start from Row's equations of motion but retain all terms linear in the ground state correlation coefficients $\kappa$ in addition to those of the RPA, which appear in the ground state expectation of the operator equations. The complete theory is discussed in Ref. 3. It should provide a convenient starting point for a semiempirical theory of the excited states of large conjugated molecules.

The purpose of the present paper is to derive and apply these higher RPA equations to the simple model system of two $\pi$ electrons in the double bond of ethylene. We choose this simple system not only because it is a model for $\pi$-electron systems, but also to show clearly how our equations are derived. This is convenient in view of the fact that the notation and concepts are not widely used. We use the same model as Ball and McLachlan ${ }^{4}$ used in their comparison of the timedependent Hartree-Fock and configuration interaction (CI) methods. All quantities such as the excitation energies, transition moments and correlation energies, are given as a function of the "interaction strength" parameter $g$.

\section{THEORY}

The $\pi$-electron model consists of two electrons with only two available orbitals, $\pi$ and $\pi^{*}$. In the HartreeFock (HF) ground state the two electrons are paired in the $\pi$ orbital, e.g., the $\pi$ electrons of the double bond of ethylene. In a more realistic ground state, i.e., the correlated ground state, the $\pi$ orbital is partially unfilled and the $\pi^{*}$ orbital is partially filled.

Second quantization provides a convenient method for studying this problem. Let $c_{i^{\prime}}{ }^{+}$and $c_{i}$, be the creation and annihilation operators, respectively, for an electron in spin orbital $i^{\prime}$ (primed indices are for spin orbitals; unprimed ones for orbitals). They satisfy the anticommutation relations:

$$
\begin{aligned}
c_{i^{\prime}} c_{j^{\prime}}+c_{j^{\prime}} c_{i^{\prime}} & =c_{i^{\prime}}+c_{j^{\prime}}++c_{j^{\prime}}+c_{i^{\prime}}+=0, \\
c_{i^{\prime}} c_{j^{\prime}}++c_{j^{\prime}}+c_{,^{\prime}} & =\delta_{i^{\prime} j^{\prime}} .
\end{aligned}
$$

For the $\mathrm{HF}$ ground state $\left|\mathrm{HF}^{\circ}\right\rangle$ we have

$$
\begin{aligned}
& c_{\pi *_{\alpha}}|\mathrm{HF}\rangle=c_{\pi *_{\beta}}|\mathrm{HF}\rangle=0, \\
& c_{\pi \alpha}+|\mathrm{HF}\rangle=c_{\pi \beta}+|\mathrm{HF}\rangle=0,
\end{aligned}
$$

where $\alpha$ and $\beta$ are the usual spin eigenstates. We can write the correlated ground state $|0\rangle$

$$
|0\rangle=N_{0}\left(1+\kappa c_{\pi} *_{\alpha}{ }^{+} c_{\pi}{ }^{+}{ }^{+} c_{\pi \beta} C_{\pi \alpha}\right)|\mathrm{HF}\rangle,
$$


where $V_{0}$ is a normalization constant and is a function of the correlation coefficient $\kappa$. Clearly $|\kappa|<1$. It is convenient to introduce a pair operator $C_{i j}{ }^{+}(S M)$, especially in extending the theory to larger systems. The operator $C_{i j}{ }^{+}(S M)$ satisfies the equation

$\left[\hat{\mathbf{S}}^{2}-S(S+1)\right] C_{i j}+(S M)=0 ; \quad\left[\hat{S}_{z}-M\right] C_{i j}{ }^{+}(S M)=0$,

where $\hat{\mathbf{S}}$ is the spin angular momentum operator. These operators have the following explicit forms

$$
\begin{aligned}
C_{i j}{ }^{+}(1 M) & =-c_{i \alpha}{ }^{+} c_{j \beta} & & (M=1) \\
& =(1 / \sqrt{2})\left[c_{i \alpha}{ }^{+} c_{j \alpha}-c_{i \beta}{ }^{+} c_{j \beta}\right] & & (M=0) \\
& =c_{i \beta}{ }^{+} c_{j \alpha} & & (M=-1) \\
C_{i j}{ }^{+}(00) & =(1 / \sqrt{2})\left[c_{i \alpha}{ }^{+} c_{j \alpha}+c_{i \beta}{ }^{+} c_{j \beta}\right] . & &
\end{aligned}
$$

The subindices $i, j$ specify the orbitals. In the case of the $\pi$-electron bond of ethylene, a given pair of orbitals $i, j$ uniquely determines the irreducible representation of the molecular symmetry group to which $C_{i j}{ }^{+}(S M)$ belongs since all its irreducible representations are one dimensional. With the definition

$$
C_{i j}{ }^{+}(\bar{S} \bar{M})=(-1)^{S+M} C_{i j}{ }^{+}(S-M),
$$

we have

$$
C_{i j}(S M)=(-1)^{M} C_{j i}+(S-M)=(-1)^{S} C_{j i}+(\bar{S} \bar{M}) .
$$

These operators have the following algebra

$$
\begin{aligned}
& {\left[C_{\imath j}{ }^{+}(S M), C_{k l}+\left(S^{\prime} M^{\prime}\right)\right]} \\
& \quad=(1 / \sqrt{2}) \sum_{S^{\prime \prime} M^{\prime \prime}} \bar{\alpha}\left(S M, S^{\prime} M^{\prime} ; S^{\prime \prime} M^{\prime \prime}\right) \\
& \times\left[\delta_{k j} C_{\imath l}+\left(S^{\prime \prime} M^{\prime \prime}\right)-(-1)^{\left.S+S^{\prime}+S^{\prime \prime} \delta_{i l} C_{k j}+\left(S^{\prime \prime} M^{\prime \prime}\right)\right] .}\right.
\end{aligned}
$$

The coefficients $\bar{\alpha}$ are $+1,-1$, or 0 and are listed in Table I.

The Hamiltonian in the second quantized form is

$$
\begin{aligned}
H=\sum_{i^{\prime}} \epsilon_{i^{\prime}} c_{i^{\prime}}+c_{i^{\prime}}+\frac{1}{2} \sum_{i^{\prime} j^{\prime}} \sum_{k^{\prime} l} V_{i^{\prime} j^{\prime} k^{\prime} l^{\prime}} c_{j^{\prime}}{ }^{+} c_{i^{\prime}}+c_{k^{\prime}} c_{l^{\prime}} \\
-\sum_{i^{\prime} j^{\prime}} \sum_{\gamma^{\prime}}\left(V_{i^{\prime} \gamma^{\prime} j^{\prime} \gamma^{\prime}}-V_{i^{\prime} \gamma^{\prime} \gamma^{\prime} j^{\prime}}\right) c_{i^{\prime}}{ }^{+} c_{j^{\prime}},
\end{aligned}
$$

where $\gamma^{\prime}$ are hole-state spin orbitals and $i^{\prime}, j^{\prime}, k^{\prime}, l^{\prime}$ can be any spin orbital (hole or particle state). $\epsilon_{i^{\prime}}$ is the

\begin{tabular}{|c|c|c|c|}
\hline$S M$ & $S^{\prime} M^{\prime}$ & $S^{\prime \prime} M^{\prime \prime}$ & $\bar{\alpha}^{\mathrm{g}}$ \\
\hline \multirow[t]{4}{*}{11} & 10 & 11 & - \\
\hline & $1-1$ & 10 & - \\
\hline & & 00 & - \\
\hline & 00 & 11 & + \\
\hline \multirow[t]{6}{*}{10} & 11 & 11 & + \\
\hline & 10 & 10 & 0 \\
\hline & & 00 & + \\
\hline & $1-1$ & $1-1$ & - \\
\hline & 00 & 10 & + \\
\hline & & 00 & 0 \\
\hline \multirow[t]{4}{*}{$1-1$} & 11 & 10 & + \\
\hline & & 00 & - \\
\hline & 10 & $1-1$ & + \\
\hline & 00 & $1-1$ & + \\
\hline \multirow[t]{6}{*}{00} & 11 & 11 & + \\
\hline & 10 & 10 & + \\
\hline & & 00 & 0 \\
\hline & $1-1$ & $1-1$ & + \\
\hline & 00 & 10 & 0 \\
\hline & & 00 & + \\
\hline
\end{tabular}
eigenvalue of the HF Hamiltonian and

$$
V_{i^{\prime} j^{\prime} k^{\prime} l^{\prime}} \equiv\left\langle i^{\prime}(1) j^{\prime}(2)\left|r_{12} 2^{-1}\right| k^{\prime}(1) l^{\prime}(2)\right\rangle .
$$

In terms of the operators $C_{i j}{ }^{+}(S M)$ we can write $H$ as

$$
\begin{aligned}
H= & \sum_{i} \epsilon_{i} \sqrt{ } 2 C_{i_{\imath}}{ }^{+}(00) \\
+ & \sum_{i j}\left\{-\frac{1}{2} \sum_{k} V_{i k k j}+\sum_{\gamma}\left(V_{i \gamma \gamma j}-2 V_{i \gamma j \gamma}\right)\right\} \sqrt{2} C_{i j}+(00) \\
& +\sum_{i j k l} V_{i j k l} C_{i k}+(00) C_{j l}+(00) .
\end{aligned}
$$

$V_{i j k l}$ are the integrals defined by Eq. (8b) but now only over spatial orbitals.
Table I. Coefficients, $\bar{\alpha}$, of Eq. (7).

All other cases give $\bar{\alpha}=0$.

\section{THE EQUATION OF MOTION AND THE HIGHER RPA}

Consider the excited states of this model system. These are just the singlet $(V)$ and triplet $(T)$ states of the $\left(\pi, \pi^{*}\right)$ configuration. From the form of $|0\rangle$, Eq. (3), we see that the operator $O^{+}(S M)$ generates an excited state of the system when acting on the ground state if

$$
O^{+}(S M)=Y(S) C_{\pi_{\pi}}+(S M)-Z(S) C_{\pi *_{\pi}}(\bar{S} \bar{M})
$$

such a state is a single-particle $\left(\pi^{*}\right)$-hole $(\pi)$ state and $\mathrm{O}^{+}(S M)$ just contains single particle-hole operators. In this model this is the only choice of $O^{+}$for states of this symmetry, but in the general case the choice of $O^{+}(S M)$ of this form, Eq. (10), is an approximation, although a very good one. The operator $O_{\lambda}{ }^{+}$for creating an excited state $|\lambda\rangle$ from $|0\rangle$ can also include two-particle-hole operators. The coefficients $Y$ and $Z$ depend on $S$ but not on $M$. From the form of $|0\rangle$ we expect $Y$ to be larger than $Z$. If we set $|0\rangle=|\mathrm{HF}\rangle$, then we have only the first term in Eq. (10). $Y$ and $Z$ are implicit functions of $\kappa$, the correlation coefficient.

To find the relation between $Y, Z$ and $\kappa$ we must solve the equation

$$
O(S M)|0\rangle=0, \quad \text { all } S, M .
$$

This equation just defines the ground state if $O^{+}(S M)|0\rangle$ is an excited state of $|0\rangle$. Noting that

$$
C_{\pi *_{\alpha}}{ }^{+} C_{\pi *_{\beta}}{ }^{+} C_{\pi \beta} C_{\pi \alpha}=C_{\pi *_{\pi}}+(00) C_{\pi *_{\pi}}{ }^{+}(00),
$$


we obtain from Eq. (11)

$$
\left[\kappa Y^{*}(S)-Z^{*}(S)\right] C_{\pi^{*} \pi}+(\bar{S} \bar{M})|\mathrm{HF}\rangle=0
$$

and finally

$$
{ }_{\kappa} Y^{*}(S)-Z^{*}(S)=0 .
$$

For normalization we require

$$
\begin{aligned}
& \left\langle 0\left|\left[O(S M), O^{+}(S M)\right]\right| 0\right\rangle \\
& \quad=\left\langle 0\left|O(S M) O^{+}(S M)\right| 0\right\rangle=1,
\end{aligned}
$$

and hence,

$$
\begin{aligned}
{\left[|Y(S)|^{2}\right.} & \left.-|Z(S)|^{2}\right] \\
& \times\left\langle 0\left|\left[C_{\pi^{*} \pi}(S M), C_{\pi^{*} \pi}+(S M)\right]\right| 0\right\rangle=1 .
\end{aligned}
$$

Recently Rowe $\mathrm{e}^{1}$ has shown by equations of motion and by variational methods that the operator $\mathrm{O}_{\lambda}{ }^{+}$for creating an excited state $|\lambda\rangle$ from the ground state is a solution of the equation ${ }^{5}$

$$
\left\langle 0\left|\left[\delta O_{\lambda}, H, O_{\lambda}{ }^{+}\right]\right| 0\right\rangle=\omega_{\lambda}\left\langle 0\left|\left[\delta O_{\lambda}, O_{\lambda}{ }^{+}\right]\right| 0\right\rangle
$$

for all variations $\delta O_{\lambda}$ and where $\omega_{\lambda}$ is the excitation energy $E_{\lambda}-E_{0}(\hbar=1)$. The double commutator is defined by

$2\left[\delta O_{\lambda}, H, O_{\lambda}{ }^{+}\right]=\left[\delta O_{\lambda},\left[H, O_{\lambda}{ }^{+}\right]\right]+\left[\left[\delta O_{\lambda}, H\right], O_{\lambda}{ }^{+}\right]$.

With $O^{+}(S M)$ of this model, Eq. (17) gives the equations

$$
\begin{gathered}
A(S) Y(S)+B(S) Z(S)=\omega(S) D Y(S), \\
A^{*}(S) Z(S)+B^{*}(S) Y(S)=-\omega(S) D^{*} Z(S),
\end{gathered}
$$

or in matrix form

$$
\begin{array}{r}
{\left[\begin{array}{cc}
A(S) & B(S) \\
-B^{*}(S) & -A^{*}(S)
\end{array}\right]\left[\begin{array}{c}
Y(S) \\
Z(S)
\end{array}\right]} \\
=\omega(S)\left[\begin{array}{cc}
D & 0 \\
0 & D^{*}
\end{array}\right]\left[\begin{array}{l}
Y(S) \\
Z(S)
\end{array}\right],
\end{array}
$$

where

$$
\begin{aligned}
A(S) & \equiv\left\langle 0\left|\left[C_{\pi^{*} \pi}(S M), H, C_{\pi^{*} \pi}+(S M)\right]\right| 0\right\rangle, \\
B(S) & \equiv-\left\langle 0\left|\left[C_{\pi^{*} \pi}(S M), H, C_{\pi^{*}}(\bar{S} \bar{M})\right]\right| 0\right\rangle, \\
D & \equiv\left\langle 0\left|\left[C_{\pi^{*} \pi}(S M), C_{\pi^{*}}+(S M)\right]\right| 0\right\rangle .
\end{aligned}
$$

Substitution of Eq. (3) for $|0\rangle$ and Eq. (9) for $H$ into Eq. (20) gives

$$
\begin{aligned}
A(S) & =N_{0}{ }^{2}\left(A^{(0)}(S)-2 \kappa K_{\pi \pi *}\right. \\
\left.\cdots \kappa^{2}\left\{A^{(0)}(S)+2\left[X-(-1)^{S} K_{\pi \pi^{*}}\right]\right\}\right), & \\
B(S)= & N_{0}{ }^{2}\left\{B^{(0)}(S)+2 \kappa\left[X-(-1)^{S} K_{\pi \pi^{*}}\right]+\kappa^{2} B^{(1)}(S)\right\}, \\
D= & N_{0}{ }^{2}\left(1-\kappa^{2}\right)=\left(1-\kappa^{2}\right) /\left(1+\kappa^{2}\right),
\end{aligned}
$$

where

$$
\begin{aligned}
A^{(0)}(S) & \equiv\left\langle\mathrm{HF}\left|\left[C_{\pi^{*} \pi}(S M), H, C_{\pi^{*} \pi}+(S M)\right]\right| \mathrm{HF}\right\rangle \\
& =\epsilon_{\pi *}-\epsilon_{\pi}+\left[1+(-1)^{S}\right] K_{\pi \pi^{*}}-J_{\pi \pi^{*}}, \\
B^{(0)}(S) & \equiv-\left\langle\mathrm{HF}\left|\left[C_{\pi *_{\pi}}(S M), H, C_{\pi *_{\pi}}(\bar{S} \bar{M})\right]\right| \mathrm{HF}\right\rangle \\
& =K_{\pi \pi^{*}}
\end{aligned}
$$

and

$$
X=\frac{1}{2}\left(J_{\pi \pi}+J_{\pi *_{\pi} *}-2 J_{\pi \pi *}\right) .
$$

$J$ and $K$ are the usual Coulomb and exchange integrals. Substitution of Eq. (21) into Eq. (19b) gives

$\left[\begin{array}{cc}\bar{A}(S) & \bar{B}(S) \\ -\bar{B}^{*}(S) & -\bar{A}^{*}(S)\end{array}\right]\left[\begin{array}{c}\bar{Y}(S) \\ \bar{Z}(S)\end{array}\right]=\omega(S)\left[\begin{array}{c}\bar{Y}(S) \\ \bar{Z}(S)\end{array}\right]$,

where

$\left[\begin{array}{cc}\bar{A}(S) & \bar{B}(S) \\ -\bar{B}^{*}(S) & -\bar{A}^{*}(S)\end{array}\right]$

$=\left[\begin{array}{cc}D^{-1 / 2} & 0 \\ 0 & D^{-1 / 2}\end{array}\right]\left[\begin{array}{cc}A(S) & B(S) \\ -\bar{B}^{*}(S) & -\bar{A}^{*}(S)\end{array}\right]\left[\begin{array}{cc}D^{-1 / 2} & 0 \\ 0 & D^{-1 / 2}\end{array}\right]$,

$$
\left[\begin{array}{c}
\bar{Y}(S) \\
\bar{Z}(S)
\end{array}\right]=\left[\begin{array}{cc}
D^{1 / 2} & 0 \\
0 & D^{1 / 2}
\end{array}\right]\left[\begin{array}{c}
Y(S) \\
Z(S)
\end{array}\right],
$$

and

$$
\begin{aligned}
\bar{A}(S) & =A(S) / D \\
& =A^{(0)}(S) \\
& -2 \kappa\left\{K_{\pi \pi *}-\kappa\left[(-1)^{S} K_{\pi \pi *}-X\right]\right\} /\left(1-\kappa^{2}\right), \\
\bar{B}(S) & =B(S) / D \\
& =B^{(0)}(S) \\
& -2 \kappa\left\{\left[(-1)^{S} K_{\pi \pi^{*}}-X\right]+\kappa K_{\pi \pi *}\right\} /\left(1-\kappa^{2}\right) .
\end{aligned}
$$

From Eq. (16) we also have

$$
|\bar{Y}(S)|^{2}-|\bar{Z}(S)|^{2}=1 .
$$

If we approximate the ground state wavefunction $|0\rangle$ in Eq. (20) by the HF function, | HF $\rangle$, Eqs. (26) and (24) are just the equations of the RPA. Note that Eq. (24) has the same form as the equations of the ordinary RPA, but this equation is still exact in the limited sense of our model system. In the RPA one neglects all terms in Eqs. (26) which depend on $\kappa$. This approximation in solving the equation of motion, Eq. (17), can be justified by the observation ${ }^{1}$ that Eq. (17) resembles an energy-weighted sum rule and should therefore be insensitive to the approximation to $|0\rangle$. But we have seen ${ }^{2}$ that for applications to molecular electronic spectra this can introduce some serious errors mainly in connection with low-lying triplet states, e.g., $N \rightarrow T$ transition of ethylene. To solve Eq. (24) we 
must know $\kappa$ which in turns means that we must solve Eq. (14). Equation (14) contains $\bar{Y}$ and $\bar{Z}$. In general an iterative procedure may be necessary. We will see that by retaining terms linear in $\kappa$ we can obtain an approximate but simple set of equations for $\omega(S)$. For a realistic system it is extremely difficult to obtain a set of Eqs. (26), accurate to all orders in $\kappa$, the correlation coefficient. We will then propose an approximation to these equations, i.e., a higher RPA.

\section{EXACT SOLUTION OF THE EQUATION OF MOTION}

Obviously Eq. (24) gives the same solution for $\omega(S)$ as the configuration interaction method. We have

$$
\begin{aligned}
\omega^{2}(S)=\bar{A}^{2}(S) & -\bar{B}^{2}(S) \\
=\omega_{0}^{2}(S) & -\left[4 \kappa /\left(1-\kappa^{2}\right)\right] \\
& \times W\left\{K_{\pi \pi^{*}}-\kappa\left[(-1)^{S} K_{\pi \pi^{*}}-X\right]\right\},
\end{aligned}
$$

where

$$
\begin{gathered}
W=\epsilon_{\pi *}-\epsilon_{\pi}+K_{\pi \pi^{*}}-J_{\pi \pi *}+X, \\
\omega_{0}(S)=\left[A^{(0) 2}(S)-B^{(0) 2}(S)\right]^{1 / 2} .
\end{gathered}
$$

In Eq. (29), $\omega_{0}(S)$ is the excitation energy in the usual RPA. We also have

$$
\begin{aligned}
\kappa & =Z(S) / Y(S)=\bar{Z}(S) / \bar{Y}(S) \\
& =-[\bar{A}(S) / \bar{B}(S)]+\left\{[\bar{A}(S) / \bar{B}(S)]^{2}-1\right\}^{1 / 2} \\
& =[\omega(S)-\bar{A}(S)] / \bar{B}(S) .
\end{aligned}
$$

Solving for $\kappa$

$$
\kappa_{ \pm}=\left(W / K_{\pi \pi^{*}}\right) \pm\left[\left(W / K_{\pi \pi^{*}}\right)^{2}+1\right]^{1 / 2} .
$$

The solution $\kappa_{-}$corresponds to the ground state. Finally

$$
\begin{aligned}
& \omega(S)=\left[\left(W^{2}+K_{\pi \pi *^{2}}\right)^{1 / 2}-X\right]+(-1)^{S} K_{\pi \pi *}, \\
& \bar{Y}(S)=1 /\left(1-\kappa^{2}\right)^{1 / 2} \text { and } \bar{Z}(S)=\kappa /\left(1-\kappa^{2}\right)^{1 / 2} .
\end{aligned}
$$

This exact solution of the equation of motion is just equivalent to the CI result. Note that the ratio of the components $\bar{Y}(S)$ and $\bar{Z}(S)$ is independent of $S$ [see Eq. (30)]. This is important and we will require this condition to hold in our higher RPA.

\section{PROPOSED HIGHER RPA}

In a realistic situation it is extremely difficult to obtain analytical expressions for $\bar{A}(S)$ and $\bar{B}(S)$ exact to all orders in $\kappa$. In our proposed treatment we will calculate $\bar{A}(S)$ and $\bar{B}(S)$ to first order in $\kappa$. Their analytical expressions are not simple but the algebraic relation Eq. (7) is very useful. We can then solve the equations for $Y(S), Z(S)$, and $\kappa$ iteratively.

To first order in $\kappa$ we have

$$
\begin{aligned}
& \tilde{A}(S)=A^{(0)}(S)-2 \kappa K_{\pi * *}, \\
& \tilde{B}(S)=B^{(0)}(S)-2 \kappa\left[(-1)^{S} K_{\pi * *}-X\right],
\end{aligned}
$$

and hence,

$$
\begin{aligned}
\ddot{\omega}^{2}(S)= & \omega^{2}(S)-4 \kappa V K_{\pi \pi^{*}} \\
& +4 \kappa^{2}\left\{K_{\pi \pi^{*}}^{2}-\left[(-1)^{S} K_{\pi \pi^{*}}-X\right]^{2}\right\} .
\end{aligned}
$$

To determine $k$ we again use Eq. (14) which requires $\tilde{Z}(S) / \tilde{Y}(S)$ to be independent of $S$, i.e., a unique ground state. From Eq. (30) we get

$$
-2 \kappa^{3}\left[(-1)^{S} K_{\pi \pi *}-X\right]-3 \kappa^{2} K_{\pi \pi *}+2 \kappa W+K_{\pi \pi^{*}}=0 .
$$

One can find the acceptable solution of this equation, i.e., $|\kappa|<1$, but note that we have one solution for $S=0$ (singlet) and another for $S=1$ (triplet). But $k$ should not depend on $S$. It does in Eq. (35), because of the truncation of the higher-order terms in $\kappa$. We will set $\kappa$ equal to the average of the two solutions, i.e., $\tilde{\kappa}=\frac{1}{2}[\tilde{\kappa}(S=0)+\tilde{\kappa}(S=1)]$. We will see that this is a good approximation.

For reasonable values for the molecular integrals, $\omega_{0}{ }^{2}(1)$ becomes negative leading to an imaginary frequency for the transition to the triplet $\left(\pi, \pi^{*}\right)$ state in the RPA. ${ }^{2}$ The linear term in $\kappa$ in Eq. (34) makes $\tilde{\omega}^{2}(1)>0$. This is readily seen by substituting the approximate solution of Eq. (35) $\kappa \approx-\frac{1}{2} K_{\pi \pi^{*}} / W$ into Eq. (34). In the next section we will apply these results to the model of Ball and McLachlan.

\section{ON THE MODEL DISCUSSED BY BALL AND MCLACHLAN}

Ball and McLachlan ${ }^{4}$ studied the singlet and triplet excited states of a model system for the $\pi$ electrons of ethylene by the time-dependent HF theory (same as the RPA). In their model the results depend on a dimensionless parameter, $g$, which describes the strength of electron correlation effects. They compared their results with those of the CI method. We now use this model to compare our proposed higher RPA with both the RPA and CI methods.

To introduce the model note that

$$
\begin{aligned}
\epsilon_{\pi *} & =I_{\pi *}+2 J_{\pi \pi *}-K_{\pi \pi *}, \\
\epsilon_{\pi} & =I_{\pi}+J_{\pi \pi},
\end{aligned}
$$

where $I_{j}$ is the matrix element of the kinetic and potential energy. To simplify the theory assume $J_{\pi \pi}=J_{\pi \pi^{*}}=J_{\pi \pi^{*}}$ and define the interaction strength

$$
g=2 K_{\pi *} /\left(I_{\pi *}-I_{\pi}\right) .
$$

The unit of energy is $|\beta|=\frac{1}{2}\left(I_{\pi^{*}}-I_{\pi}\right)$. With these assumptions we have

$$
\begin{gathered}
X=0 ; \quad W=2|\beta|, \\
A^{(1)}(S)=\left[2+(-1)^{S} g\right]|\beta|, \\
B^{(0)}(S)=g|\beta|, \\
\bar{A}(S)=\left[2+(-1)^{S} \bar{g}(S)\right]|\beta|, \\
\bar{B}(S)=\bar{g}(S)|\beta|,
\end{gathered}
$$


where

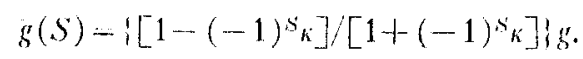

The usual RPA solutions are

$$
\begin{aligned}
& \omega_{0}(S)=2\left[1+(-1)^{S} g\right]^{1 / 2}|\beta|, \\
& Y_{0}(S)=\left\{1+\left[1+(-1)^{S} g\right]^{1 / 2}\right\} / 2\left[1+(-1)^{S} g\right]^{1 / 4}, \quad(42 \mathrm{~b}) \\
& Z_{10}(S)=(-1)^{S}\left\{1-\left[1+(-1)^{S} g\right]^{1 / 2}\right\} / 2\left[1+(-1)^{S} g\right]^{1 / 4} .
\end{aligned}
$$

The exact solutions of the equation of motion, i.e., $\omega(S), \bar{Y}(S)$, and $\bar{Z}(S)$, have the same form as the RPA solutions with $g$ replaced by $\bar{g}$. Similarly the solutions for our higher RPA are obtained from those of the RPA by replacing $g$ with

$$
\hat{g}(S)=\left[1-(-1)^{s} 2 \kappa\right] g .
$$

We now consider the excitation frequency in the various approximations: (i) $\omega(S)$, the exact solution to the model, (ii) $\tilde{\omega}(S)$, the proposed approximation to the exact equation of motion, (iii) the RPA. These will all be expressed in a power series in $g$, the interaction strength. For the range $0 \leq \frac{1}{2} g<1$ we have

$$
\omega(S) /|\beta|=2+(-1)^{S} g+\left(\frac{1}{2} g\right)^{2}-\left(\frac{1}{2} g\right)^{4}+\cdots
$$

and

$\tilde{\omega}(S) /|\beta|=2+(-1)^{S} g+\left(\frac{1}{2} g\right)^{2}-(-1)^{S}\left(\frac{1}{2} g\right)^{3}+\cdots$.

The RPA solution $\omega_{0}$ becomes unstable for $g \geq 1$. The excitation frequency in the higher RPA, $\bar{\omega}(S)$, does not become unstable at $g \geq 1$. In the region $0 \leq g<1$ we have for the RPA

$$
\omega_{0}(S) /|\beta|=2+(-1)^{S} g-\left(\frac{1}{2} g\right)^{2}+\cdots .
$$

The HF estimate of the frequency $\omega_{\mathrm{HF}}$ is

$$
\omega_{\mathrm{HF}}(S) /|\beta|=2+(-1)^{S} g .
$$

From Eqs. (44)-(47) we see that the RPA or timedependent $\mathrm{HF}$ theory incorrectly predicts an excitation frequency smaller than the HF value, Eq. (47). The true excitation frequency $\omega(S)$ is larger than the HF estimate, $\omega_{\mathrm{HF}}$. In this particular model the exact solution should include no correlation in the excited states since there is only one particle state. It is a poor model for studying the effects on the excitation frequency of the correlations included in the RPA. The correlation coefficient $\kappa$ from the exact solution Eq. (31) is

$\kappa=-\frac{1}{2}\left(\frac{1}{2} g\right)+\frac{1}{8}\left(\frac{1}{2} g\right)^{3}-\frac{1}{16}\left(\frac{1}{2} g\right)^{5}+\cdots, \quad 0 \leq g<2$.

The approximation $\tilde{\kappa}(S)$ from the higher RPA is

$$
\tilde{\kappa}(S)=-\frac{1}{2}\left(\frac{1}{2} g\right)+\frac{3}{8}\left(\frac{1}{2} g\right)^{3}-(-1)^{S \frac{1}{8}\left(\frac{1}{2} g\right)^{4}+\cdots .}
$$

For a unique ground state $\tilde{\kappa}(S)$ should be independent of $S$, i.e., Eq. (11) must have a unique solution. Note that for our approximation the dependence on $S$ comes in at $O\left(g^{4}\right)$. For the RPA we obtain

$$
\kappa_{0}(S)=-\frac{1}{2}\left(\frac{1}{2} g\right)+(-1)^{S \frac{1}{2}\left(\frac{1}{2} g\right)^{2}-\frac{1}{8}\left(\frac{1}{2} g\right)^{3}+\cdots .}
$$

In the RPA the correlation coefficient $\kappa_{0}$ depends on $S$, i.e., is nonunique, at $O\left(g^{2}\right)$. For ethylene, $g=0.530^{\circ}$, and we have the exact $\kappa=-0.1321, \tilde{\kappa}(S=0) \approx \tilde{\kappa}(S=1) \approx$ -0.128 , and $\left.\kappa_{0}(S=0) \cong-0.099, \quad \kappa_{0}(S=1) \cong-1\right) .171$. Clearly in the RPA, the ground state as determiner by the criterion $O(S=0)|0\rangle=0$ is quite different from that determined by $O(S=1)|0\rangle=0$.

\section{THE CORRELATION ENERGY}

The correlation energy is defined as the difference between the HF energy and the exact energy. With the function $|0\rangle$ of $\mathrm{Eq}$. (3) we have

$$
I_{\mathrm{cerr} r \mathrm{r}}=\left[\kappa /\left(1+\kappa^{2}\right)\right]\left\{2 K_{\pi \pi *}+\kappa\left(H_{\pi *}-E_{\mathrm{HF}}\right)\right\},
$$

where

$$
\begin{aligned}
& H_{I I V}=2 \epsilon_{\pi}-J_{\pi \pi}, \\
& H_{\pi *}=2 \epsilon_{\pi *}+J_{\pi \pi^{*}}-2\left(\beta J_{\pi \pi^{*}}-K_{\pi \pi^{*}}\right) .
\end{aligned}
$$

With $\kappa$ of Eq. (48) and $0 \leq g<2$

$$
E_{\text {corr }} /|\beta|=-\left(\frac{1}{2} g\right)^{2}+\frac{1}{4}\left(\frac{1}{2} g\right)^{4}-\frac{1}{8}\left(\frac{1}{2} g\right)^{6}+\cdots .
$$

With $\tilde{\kappa}$ of the proposed higher RPA we obtain

$\tilde{E}_{\text {corr } /} / \beta \mid=-\left(\frac{1}{2} g\right)^{2}+\frac{1}{4}\left(\frac{1}{2} g\right)^{4}-\frac{1}{8}\left(\frac{1}{2} g\right)^{6}+\cdots, \quad 0 \leq g<2$.

For small $g$

$$
\left(E_{\text {corr }}-\widetilde{E}_{\text {corr }}\right) /|\beta| \approx_{\frac{1}{4}\left(\frac{1}{2} g\right)^{6}} .
$$

This result suggests that the proposed higher RPA may be a very useful method for obtaining estimates of the correlation energy in closed-shell systems. With the $\kappa_{0}$ of the usual RPA we obtain

$$
F_{\mathrm{ourr}} \mathrm{RPA} /|\beta|=-\left(\frac{1}{2} g\right)^{2}+\frac{5}{4}\left(\frac{1}{2} g\right)^{4}+\cdots .
$$

The effect of the difference in $\kappa_{0}$ derived from the singlet and triplet operators $O^{+}(S M)$ on $E_{\text {corr }}{ }^{\text {RPA }}$ appears in $O\left(g^{5}\right)$.

Ball and Mclachlan ${ }^{4}$ obtained an approximate expression for the correlation energy which, for this model, gives

$$
E_{\text {corr }}{ }^{\mathrm{TDHF}} /|\beta|=-\left(\frac{1}{2} g\right)^{2}-\frac{1}{2}\left(\frac{1}{2} g\right)^{3}-\frac{5}{4}\left(\frac{1}{2} g\right)^{4}-\cdots .
$$

Comparison with Eq. (53) shows that this expression overestimates the ground state correlation energy.

\section{ONE- AND TWO-PARTICLE DENSITIES}

We now examine the one- and two-particle densities for this model. The one- and two-particle density operators $\hat{\rho}_{i^{\prime} j^{\prime}}$ and $\hat{\rho}_{l^{\prime} j^{\prime}: k^{\prime} l^{\prime}}$ are

$$
\begin{aligned}
\hat{\rho}_{i^{\prime} j^{\prime}} & =c_{i^{\prime}}{ }^{+} c_{j^{\prime}}, \\
\hat{\rho}_{i^{\prime} j^{\prime}: k^{\prime} l^{\prime}} & =c_{j^{\prime}}+{ }^{+} c_{i^{\prime}}+c_{k^{\prime}} c_{l^{\prime}},
\end{aligned}
$$

respectively. In terms of these operators we can write the matrix element of any one- and two-particle operator between the many-electron states $V$ and $W$ as

$$
\begin{aligned}
& F_{V W}=\sum_{i^{\prime} j^{\prime}} F_{i^{\prime} j^{\prime}}\left(\rho_{i^{\prime} j^{\prime}}\right)_{V W}, \\
& G_{V W}=\sum_{i^{\prime} j^{\prime}} \sum_{k^{\prime} l^{\prime}} G_{i^{\prime} j^{\prime} k^{\prime} l^{\prime}}\left(\rho_{i^{\prime} j^{\prime}: k^{\prime} l^{\prime}}\right)_{V W} .
\end{aligned}
$$


The pair correlation function in the ground state, $\left(\rho_{a b: c d}\right)_{00}$, can be written in terms of matrix elements of $\hat{\rho}_{i^{\prime} j^{\prime}}$ :

$$
\left(\rho_{a b: c d}\right)_{00}=\rho_{a c} \rho_{b d}-\delta_{a b} \rho_{b c}+\sum_{n \neq 0}\left(\rho_{a c}\right)_{0 n}\left(\rho_{b d}\right)_{n 0},
$$

where, for instance, $\rho_{a c} \equiv\left(\rho_{a c}\right)_{00}$. We now let the spin orbitals $a=a \alpha$ and $a^{\prime}=\alpha \beta$ denote the atomic orbitals on the two atoms. In this model, orbitals $a$ and $b$ are assumed orthogonal, i.e.,

$$
\left.\pi_{\pi}^{*}\right\}=(1 / \sqrt{2})(a \mp b) .
$$

By symmetry, $\rho_{a a}=\rho_{b b}=\frac{1}{2}$. Also $\left(\rho_{a a}\right)_{01}=-\left(\rho_{b b}\right)_{01}$, $\left(\rho_{a a}\right)_{03}=-\left(\rho_{b b}\right)_{03}$, and

$$
\begin{aligned}
& \left(\rho_{a a}\right)_{01}=(1 / 2 \sqrt{2})[Y(0)+Z(0)] D, \\
& \left(\rho_{a a}\right)_{03}=(1 / 2 \sqrt{2})[Y(1)-Z(1)] D,
\end{aligned}
$$

where $D$ is given in Eq. (20). For the RPA or TDHF we set $D=1$ and use the RPA solutions for $Y(S)$ and $Z(S)$. With these elements of the one-particle density matrix we evaluate various components of the pair correlation function. With $g=0.539$ for ethylene the TDHF gives $^{4}$

$$
\begin{aligned}
\rho_{a b^{\prime}: a b^{\prime}} & =0.333, \\
\rho_{a b: a b} & =-0.035, \\
\rho_{a a^{\prime}: a a^{\prime}} & =0.167, \\
\rho_{a a: a a} & =0.035,
\end{aligned}
$$

and the higher RPA:

$$
\begin{aligned}
\rho_{a b^{\prime}: a b^{\prime}} & =0.312, \\
\rho_{a b: a b} & =0, \\
\rho_{a a^{\prime}: a a^{\prime}} & =0.187, \\
\rho_{a a: a a} & =\mathbf{0},
\end{aligned}
$$

while the CI values are $0.315,0,0.185,0$, respectively. The higher RPA provides good estimates for the components of the two-particle density matrix. In a larger system this can be a significant advantage since the higher RPA solution requires considerably less effort than the direct CI solution.

\section{TRANSITION MATRIX ELEMENTS}

Next we consider the transition matrix elements for both the singlet and triplet excited state of this model. For singlet and triplet transitions we need matrix elements of the operators $q$ and $\sigma$, respectively, where in second quantized form and for nonoverlapping atomic orbitals

$$
\begin{aligned}
& \mathbf{q}=(\mathbf{R} / \sqrt{2})\left[C_{\pi \pi_{\pi}}+(00)+C_{\pi \pi *^{+}}(00)\right], \\
& \boldsymbol{\sigma}=(\mathbf{R} / \sqrt{2})\left[C_{\pi * \pi}+(10)+C_{\pi \pi *^{+}}(10)\right] .
\end{aligned}
$$

$\mathbf{R}$ is the bond length vector. For this model $\left\langle\pi|\mathbf{q}| \pi^{*}\right\rangle=$ $\frac{1}{2} R$ where $\mathbf{q}$ is the coordinate vector of the electron. The transition elements of interest are

$$
\begin{aligned}
& \langle 0|\mathbf{q}| 1\rangle=(\mathbf{q})_{01}=\left\langle 0\left|\left[\mathbf{q}, O^{+}(00)\right]\right| 0\right\rangle, \\
& \langle 0|\boldsymbol{\sigma}| 3\rangle=(\boldsymbol{\sigma})_{03}=\left\langle 0\left|\left[\boldsymbol{\sigma}, O^{+}(10)\right]\right| 0\right\rangle,
\end{aligned}
$$

in obvious notation.

Denote the squared transition moments by $q^{2}$ and $\sigma^{2}$. Expanded in powers of $g$ the TDHF (also the RPA) ${ }^{4}$ gives for $g<1$

$$
\begin{aligned}
& q_{0}{ }^{2}=\frac{1}{2} R^{2}\left\{1-\left(\frac{1}{2} g\right)+\frac{3}{2}\left(\frac{1}{2} g\right)^{2}-\frac{5}{2}\left(\frac{1}{2} g\right)^{3}+\cdots\right\}, \\
& \sigma_{0}{ }^{2}=\frac{1}{2} R^{2}\left\{1+\left(\frac{1}{2} g\right)+\frac{3}{2}\left(\frac{1}{2} g\right)^{2}+\frac{5}{2}\left(\frac{1}{2} g\right)^{3}+\cdots\right\},
\end{aligned}
$$

and the CI solution for $g<2$

$$
\begin{aligned}
& q^{2}=\frac{1}{2} R^{2}\left\{1-\left(\frac{1}{2} g\right)+\frac{1}{2}\left(\frac{1}{2} g\right)^{3}-\frac{3}{8}\left(\frac{1}{2} g\right)^{5}+\cdots\right\}, \\
& \sigma^{2}=\frac{1}{2} R^{2}\left\{1+\left(\frac{1}{2} g\right)-\frac{1}{2}\left(\frac{1}{2} g\right)^{3}+\frac{3}{8}\left(\frac{1}{2} g\right)^{5}-\cdots\right\} .
\end{aligned}
$$

The higher RPA, gives for $g<2$

$$
\begin{aligned}
& \tilde{q}^{2}=\frac{1}{2} R^{2}\left\{1-\left(\frac{1}{2} g\right)+\frac{3}{4}\left(\frac{1}{2} g\right)^{3}-\cdots\right\}, \\
& \tilde{\sigma}^{2}=\frac{1}{2} R^{2}\left\{1+\left(\frac{1}{2} g\right)-\frac{3}{4}\left(\frac{1}{2} g\right)^{3}+\cdots\right\} .
\end{aligned}
$$

\section{DISCUSSION}

We have applied our proposed higher-order RPA to the simple model system of two $\pi$ electrons in the double bond of ethylene. The usual RPA is the simplest approximation to the solution of the equations of motion for the excitation frequency of a transition. The method clearly underestimates the excitation energies for singlet excited states ${ }^{2}$ but runs into difficulty for some triplet excited states. Our proposed higher-order RPA removes the difficulties involved in the usual RPA but retains the form of the RPA equations. To derive the higher RPA (see Ref. 3 for a general case), we retain all terms linear in the ground-state correlation coefficients in the equations for the excitation frequency and in the equations determining the ground state. These equations are solved self-consistently. We do not use the quasiboson approximation, and in fact, we show that it is a poor approximation for atomic and molecular systems.

The RPA and the proposed higher RPA ${ }^{3}$ are approximate solutions of the equations-of-motion method for excitations. The higher RPA is a good approximation to the CI solution. Both the RPA and higher RPA violate the Pauli exclusion principle to some extent, but clearly the higher RPA is a much better approximation in this regard. We believe that this method represents an optimum approach to predicting relative quantities such as an excitation frequency and a transition moment directly since the equations are set up so as to be insensitive to the complexities of the stationary state wavefunctions. We have used the higher RPA (HRPA) to study the singlet $(V)$ and triplet $(T)$ excited states $\left(\pi, \pi^{*}\right)$ of the $\pi$ electrons of ethylene. The model is that introduced by Ball and McLachlan ${ }^{4}$ in which the interaction strength for the pair of electrons can be varied. This is a simple model, and in this way we can show many details which would otherwise be lost in applications to larger systems. The results suggest that the usual RPA underestimates the ground-state cor- 
relation energy while overestimating the correlation energy of the excited state.

Applications to larger systems will be published separately. ${ }^{6}$ The method does not run into any instabilities such as those which sometimes occur in the RPA, and it provides very reliable estimates of excitation frequencies, transition moments, pair correlation functions, and even the correlation energy. In a later publication ${ }^{6}$ we will discuss how one can develop useful approximations to the solution of the equations of the higher RPA.

\section{APPENDIX: RENORMALIZATION OF ORBITAL ENERGIES}

In this appendix we discuss certain renormalized particle energies introduced by Rowe ${ }^{1}$ in his formulation of a remormalized RPA. With this simple model the differences between these renormalized particle energies become obvious.

A detailed analysis ${ }^{1}$ shows that the matrix element

$$
A_{m \gamma n \delta}=\left\langle 0\left|\left[c_{m}{ }^{+} c_{\gamma}, H, c_{n}{ }^{+} c_{\delta}\right]\right| 0\right\rangle
$$

contains terms such as $\left\langle 0\left|c_{m}\left[H, c_{n}{ }^{+}\right]\right| 0\right\rangle,\left\langle 0\left|c_{\gamma}{ }^{+}\left[H, c_{\delta}\right]\right| 0\right\rangle$, $\left\langle\left|\left\{c_{\gamma}{ }^{+},\left[H, c_{\delta}\right]\right\}\right|\right\rangle$, and $\left\langle\left|\left\{c_{m},\left[H, c_{n}{ }^{+}\right]\right\}\right|\right\rangle$, where |\rangle is the particle-hole vacuum. In Eq. (A1), $m$ and $n$ denote particle states, while $p, q, r, s$ represent particle or hole states. The single-particle basis must be specified. Rowe ${ }^{1}$ suggests that a convenient basis would be one which diagonalizes the matrix element $\mathcal{E}_{p}$ with

$$
\begin{aligned}
\left\langle 0\left|\left\{c_{p}\left[H, c_{q}{ }^{+}\right]\right\}\right|\right\rangle & =T_{p q}+\sum_{r s} V_{r p s q}\left\langle 0\left|c_{r}{ }^{+} c_{s}\right| 0\right\rangle \\
& =\varepsilon_{p} \delta_{p q} .
\end{aligned}
$$

This is just a generalization of the HF single-particle basis in which the single-particle field is calculated from the true rather than the HF density. To derive his renormalized RPA, Rowe $\mathrm{e}^{1}$ also assumes that this representation diagonalizes the matrix elements $\left\langle 0\left|c_{m}\left[H, c_{n}{ }^{+}\right]\right| 0\right\rangle$ and $\left\langle 0\left|c_{\gamma}+\left[H, c_{\delta}\right]\right| 0\right\rangle$ and defines new single-particle energies $\mathcal{E}^{(+)}$and $\mathcal{E}^{(-)}$, i.e.,

$$
\begin{aligned}
\left\langle 0\left|c_{m}\left[H, c_{n}{ }^{+}\right]\right| 0\right\rangle & \simeq \delta_{m n}\left\langle 0\left|c_{m}\left[H, c_{m}{ }^{+}\right]\right| 0\right\rangle \\
& \equiv \delta_{m n} \varepsilon_{m}{ }^{(+)}\left\langle 0\left|c_{m} c_{m}{ }^{+}\right| 0\right\rangle, \\
\left\langle 0\left|c_{\gamma}{ }^{+}\left[H, c_{\delta}\right]\right| 0\right\rangle & \simeq \delta_{\gamma \delta}\left\langle 0\left|c_{\gamma}{ }^{+}\left[H, c_{\delta}\right]\right| 0\right\rangle \\
& \equiv-\delta_{\gamma \delta} \varepsilon_{\gamma}{ }^{(-)}\left\langle 0\left|c_{\gamma}{ }^{+} c_{\gamma}\right| 0\right\rangle .
\end{aligned}
$$

If we use $|\mathrm{HF}\rangle$ for $|0\rangle$, these quantities are just $\mathrm{HF}$ orbital energies, $\epsilon_{i}$

$$
\varepsilon_{m}=\varepsilon_{m}{ }^{(+)}=\epsilon_{m} ; \quad \varepsilon_{\gamma}=\varepsilon_{\gamma}^{(-)}=\epsilon_{\gamma} .
$$

For this simple model with the correlated ground state of Eq. (3) we can obtain explicit expressions for these single-particle energies

$$
\begin{aligned}
\mathcal{E}_{\pi *} & =\epsilon_{\pi *}-\left[\left(2 J_{\pi \pi *}-K_{\pi \pi^{*}}\right)-J_{\pi^{*} \pi^{*}}\right]\left[\kappa^{2} /\left(1+\kappa^{2}\right)\right], \\
\mathcal{E}_{\pi} & =\epsilon_{\pi}+\left[\left(2 J_{\pi \pi^{*}}-K_{\pi \pi^{*}}\right)-J_{\pi \pi}\right]\left[\kappa^{2} /\left(1+\kappa^{2}\right)\right], \\
\mathcal{E}_{\pi^{*}}\left({ }^{+)}\right) & =\epsilon_{\pi}-\kappa K_{\pi \pi^{*},} \\
\mathcal{E}_{\pi}^{(-)} & =\epsilon_{\pi}+\kappa K_{\pi \pi^{*}} .
\end{aligned}
$$

TABLE II. Single-particle energies.

\begin{tabular}{lrr}
\hline \hline & STO basis & $\begin{array}{c}(3 s 2 p / 1 s) \\
\text { GTO basis }\end{array}$ \\
\hline$\epsilon_{r^{*}}$ & 0.2056 & 0.1355 \\
$\epsilon_{\pi}$ & -0.4047 & -0.3865 \\
$\kappa$ & -0.2558 & -0.1768 \\
$\varepsilon_{\pi^{*}}^{(+)}$ & 0.2461 & 0.1527 \\
$\varepsilon_{\pi^{*}}$ & 0.1863 & 0.1263 \\
$\mathcal{E}_{\pi^{(-)}}$ & -0.4452 & -0.4037 \\
$\mathcal{E}_{\pi}$ & -0.3846 & -0.3803 \\
& & \\
\hline
\end{tabular}

From Eq. (3), $\kappa^{2} /\left(1+\kappa^{2}\right)$ is just the number of particles occupying the $\pi^{*}$ orbital. Note that $\varepsilon_{\pi^{*}}-\epsilon_{\pi *}$ (or $\varepsilon_{\pi}-\epsilon_{\pi}$ ) is quadratic in $\kappa$ while $\mathcal{E}_{\pi^{*}}(+)-\epsilon_{\pi} *$ or $\mathcal{E}_{\pi}^{(-)}-\boldsymbol{\epsilon}_{\pi}$ ] is linear in $\kappa$. In general, $\varepsilon_{m}{ }^{(-)}-\epsilon_{m}$ and $\varepsilon_{\gamma}^{(+)}-\epsilon_{\gamma}$ are both linear in the correlation coefficients of $|0\rangle$, while $\varepsilon_{m}-\epsilon_{m}$ and $\varepsilon_{\gamma}-\epsilon_{\gamma}$ are quadratic in these coefficients, i.e., they go like particle or hole densities. This is obviously an important difference, especially if one wants to calculate excitation frequencies and ground-state correlations self-consistently. To derive his renormalized RPA Rowe ${ }^{1}$ sets $\varepsilon_{m}=\varepsilon_{m}{ }^{(+)}$and $\varepsilon_{\gamma}=\varepsilon_{\gamma}{ }^{(-)}$. From our results this is not a consistent approximation.

We now evaluate some of these single-particle energies using two approximate $\mathrm{HF}$ wavefunctions for ethylene. We consider only the $\pi$ and $\pi^{*}$ orbitals. Table II lists these results. Those in the first column are obtained using the HF orbitals from a calculation with a minimal basis set of Slater orbitals, while those in the second column are from a calculation with a set of contracted Gaussian orbitals. ${ }^{7}$ The values of $\kappa$ are those of the CI. The ionization potential of the $\pi$ electron in ethylene is $10.51 \mathrm{eV}$ or 0.3863 a.u. In the STO basis $\boldsymbol{\epsilon}_{\pi}$, Koopman's theorem estimate for the ionization potential, is 0.4047 , while $\varepsilon_{\pi}$ is -0.3846 . From its definition we expect $\varepsilon_{\gamma}$ to be an improved estimate of the ionization potential.

* Contribution No. 4053

1 D. J. Rowe, Rev. Mod. Phys. 40, 153 (1968).

2 T. H. Dunning and V. McKoy, J. Chem. Phys. 47, 1735 (1967) ; 48, 5263 (1968).

${ }^{3}$ T. Shibuya and V. McKoy, "Higher Random-Phase Approximation as an Approximation to the Equations of Motion," Phys. Rev. (to be published).

${ }^{4}$ M. A. Ball and A. D. McLachlan, Mol. Phys. 7, 501 (1964).

${ }^{5}$ We have used the Hartree atomic units throughout the paper unless otherwise indicated.

${ }^{6}$ T. Shibuya and V. McKoy, "Application of the RPA and Higher RPA to the $V$ and $T$ states of Ethylene," J. Chem. Phys. (to be published).

${ }^{7} \mathrm{~A}$ set of three $s$-like and two $p$-like Gaussian orbitals on the carbon atom obtained by contraction from a set of nine $s$-like and five $p$-like GTO. The hydrogen basis is a single contracted orbital derived from four $s$-like functions. See $N$. W. Winter, T. H. Dunning, Jr., and V. McKoy, J. Chem. Phys, 49, $4128(1968)$. 